\title{
Common Etiology of Foreign Body Ingestion
}

\author{
Adnan Qureshi ${ }^{1 *}$, Joanne Cunningham ${ }^{2}$ and Sobia $\mathrm{Naz}^{3}$ \\ ${ }^{1}$ Consultant Colorectal Surgeon, Northampton General Hospital NHS Trust, UK \\ ${ }^{2}$ Department of Cellular Pathology, Milton Keynes University Hospital NHS Trust, UK \\ ${ }^{3}$ Psych Consultant Child and Adolescent Psychiatrist, East London Mental Health, UK
}

*Corresponding author: Adnan Qureshi, Consultant General \& Colorectal Surgeon, Department of Surgery Northampton General Hospital, NN1 5BD, UK

Submission: August 01, 2018; Published: August 20, 2018

\begin{abstract}
Background: The aim of this study was to look at the demography, sites of insertion, most common causes of foreign body ingestion and the procedures used for retrieval of these foreign bodies (FB). We also looked at length of stay and common a mental health diagnosis for patients admitted with FB ingestion.
\end{abstract}

Material \& Method: This was a retrospective analysis of four years data from January 2014 to January 2018 at Northampton general Hospital. The data was collected using the hospital electronic record system. All patients admitted under surgical subspecialties and gastroenterology was included. Children with a genital FB were excluded from the study. The number of admissions for each patient was recorded. Patients were divided into accidental and mental health illness (MHI) groups.

Result: A total of 146 patients were admitted with FB diagnosis. 57\% (84) were in the accidental group and 43\% (63) were in the MHI group. In the accidental group $70 \%$ (54) were under the age of 16 years, the most common cause was inorganic FB and the most common site was an ENT. While in adults most common site in accidental FB was oesophagus and the food bolus was the commonest cause. In the MHI group 63 patients had 257 admissions episodes. The mean age was $25 \pm 21$ years. Female sex and younger age i.e early adulthood were at a higher risk for FB ingestion. Abdominal $x$-ray was the most common investigation. Mean length of stay was $3 \pm 2$ days. Endoscopic retrieval was successful in $85 \%$ of patients. Most common mental health diagnosis was deliberate self harm (DSH) $40 \%$.

Conclusion: Younger age group, female sex and patients with history of DSH are at a higher risk of FB ingestion and recurrent presentation. Early recognition of high risk patients and prompt psychiatric help can minimise the recurrent presentation of these patients. Minimally invasive techniques like endoscopic retrieval of FB should be first choice.

Keywords: Foreign body; Mental health illness; Deliberate self harm

Abbreviations: DSH: Deliberate Self Harm; PD: Personality Disorder; PTSD: Post Traumatic Stress Disorder; LD: Learning Disability

\section{Introduction}

Patients with foreign bodies (FB) are common admissions to gastroenterology and surgical wards [1,2]. Patients with severe mental illness can be admitted on to a surgical ward with a variety of presentations ranging from foreign body ingestion, subcutaneous insertion, and introduction of FBs into natural orifices like the vagina, urethra or rectum. Foreign body ingestion into the gastrointestinal tract can lead to serious consequences with significant morbidity and mortality. Eighty percent of ingested foreign bodies which reach the stomach will pass uneventfully through the gastrointestinal tract (GI). Those which can't pass through the GI track require either endoscopic or surgical intervention. It is important to understand that common mental health conditions leading to foreign body ingestion or insertion and to identify which patients are more likely to ingest complex foreign bodies.
Inedible substance ingestion increases the risk of ileus, poisoning, and suffocation and the long term consequences of ingestion of substances like detergents, acids etc are very poor. There is also a higher complication rate in patients repeatedly introducing FB under the skin or into the rectum or vagina. Complications range from damage to the surrounding structures to intestinal fistulae and peritonitis. This means prevention is important, especially in patients with mental health illnesses. Most of the patients with MHI, who are frequent attenders, are either in a psychiatric institution or under direct psychiatric observation [3]. we reviewed the data of all the patients who were admitted in our institute with a FB diagnosis which required surgical or endoscopic intervention for retrieval. We identified all the patients with a mental health illness for further analysis. 


\section{Method}

Data for all the patients admitted with a diagnosis of a FB was retrospectively analysed covering the four year period from January 2014 to January 2018. Electronic discharge summaries, online PACS system for radiological investigations and operation notes were reviewed, which include details of presentation, investigations, management and documentation of any mental health problems.

\section{Inclusion criteria}

A. All patients with a diagnosis of FB from January 2014 to January 2018.

\section{Exclusion criteria}

A. Genital FB under the age of 16 years.

The patients identified were then divided into two cohorts; those with accidental FB and those with MHI. We reviewed patient demography, a history of FB ingestion (i.e. accidental, self-harm or sexual gratification) and the most common presentation (e.g. ingestion, subcutaneous insertion etc). We also looked at common investigations for diagnosis and common methods of retrieval. The

Table 1: Common causes of foreign body insertion. length of hospital stay was recorded and for patients in the MHI cohort we reviewed any documentation of a psychiatric diagnosis and the number of repeated admissions for each patient.

\section{Result}

Over the four year study period, a total of 151 were identified with FB out of which 146 patients were admitted under surgery, urology and ENT departments. 57\% (84) of these patients were in the accidental group and 43\% (63) were in the MHI group. The common causes of FB insertion in these cohorts are shown in Table 1 the accidental cohort was further dived into paediatric and adult sub-groups. We found the majority 70\% (58) of these patients were in the paediatric age group. The male to female ratio was 1.8:1 showing a slight male predominance and the mean age at presentation was $24.5 \pm 22$ years. The most common site of foreign body insertion in the paediatric age group was the ear, nose and throat (ENT) followed by the oesophagus. In the adult group this was the oesophagus (i.e. food bolus). Table 2 shows the common sites FB in accidental group. Five paediatric patients with genital FB were admitted directly under paediatrics this was due to local child safeguarding policies.

\begin{tabular}{|c|c|}
\hline Mental Health Illness & Non Psychiatric \\
\hline Deliberate self-harm (DSH) & Accidental \\
\hline Compulsive sexual behaviour (CSB) & Sexual Gratification \\
\hline Borderline personality disorder (Eating disorders) & Dementia \\
\hline Delusion or command hallucination (Schizophrenia) & Cognitive problems \\
\hline & Sexual assault or prank \\
\hline & Drug concealment ("Body packing") \\
\hline
\end{tabular}

Table 2: Accidental foreign body $(\mathrm{n}=84)$.

\begin{tabular}{|c|c|c|c|c|}
\hline \multirow{2}{*}{ Site of Ingestion } & \multicolumn{2}{|c|}{ Paediatrics (n=59) } & \multicolumn{2}{c|}{ Adults (n=25) } \\
\cline { 2 - 5 } & Male (n=38) & Female (21) & Male (n=16) & Female (n=9) \\
\hline ENT & $22(57 \%)$ & $13(62 \%)$ & $2(13 \%)$ & $2(22 \%)$ \\
\hline GI & $10(26 \%)$ & $6(28 \%)$ & $11(69 \%)$ & $6(67 \%)$ \\
\hline Skin & $6(15 \%)$ & $1(2 \%)$ & $3(19 \%)$ & $1(11 \%)$ \\
\hline
\end{tabular}

Accidental (n=84)

Figure 1: Schematic diagram of patient distribution. 
These patients never had any surgical input so were excluded from study. In the MHI cohort among 63 patients, a total of 257 admissions for removal of a foreign body were recorded. We found the total number of procedures on individual patients ranged from 1 to 21 with a mean of more than four procedures per patient. The male to female ratio was 1:1.2 which a slight female predominance. The mean age at presentation was $25 \pm 21$ years. Figure 1 shows the overall comparison of M:F ratio in the accidental and MHI cohorts, where the male predominance in the accidental group and female predominance in the MHI group can be observed.

Figure 2 shows the age distribution comparing both cohorts. There is a higher incidence of FB insertion in the younger population in both cohorts. The incidence of FB insertion in older patients in the MHI group is very small and is limited to learning disability patients only. The FB insertion in the accidental group actually starts to increase in old age due to cognitive dysfunction. Figure 3 Shows the documented psychiatric diagnosis in medical records and compares admissions in male and female patients. We found a female predominance toward deliberate self-harm $(60 \%)$, followed by personality disorders (20\%). Other diagnoses, in female patients, included psychosis (8\%), post-traumatic stress disorder (6\%), Compulsive sexual behaviour (CSB) 6. In male patients the most prominent diagnosis was sexual gratification (50\%), followed by deliberate self-harm (17\%). Other mental health diagnosis was personality disorder (11\%), psychosis (8\%), schizophrenia (7\%) and learning disabilities (7\%).

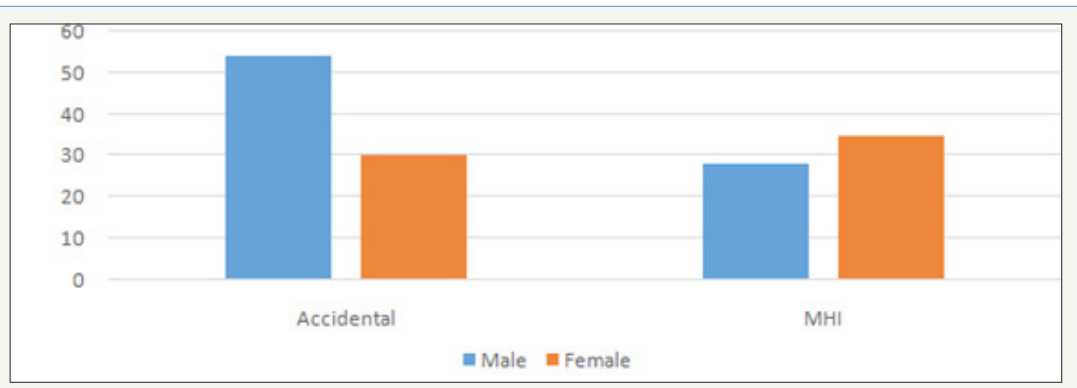

Figure 2: Male: Female Ratio $(n=146)$.

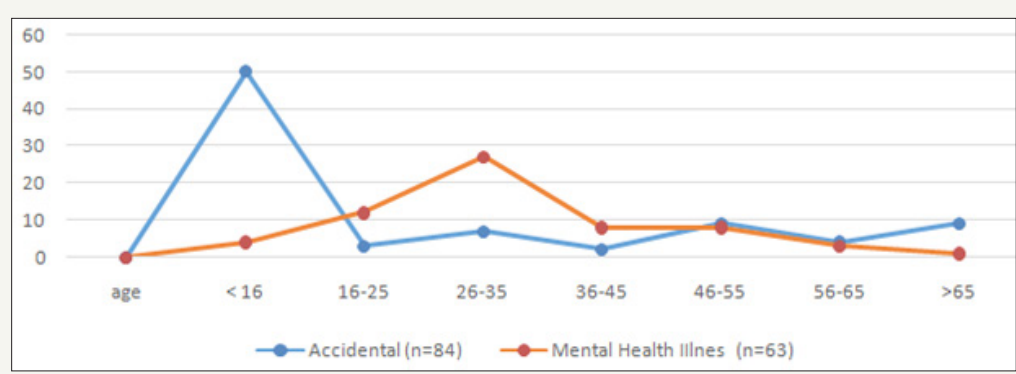

Figure 3: Age distribution $(\mathrm{n}=146)$.

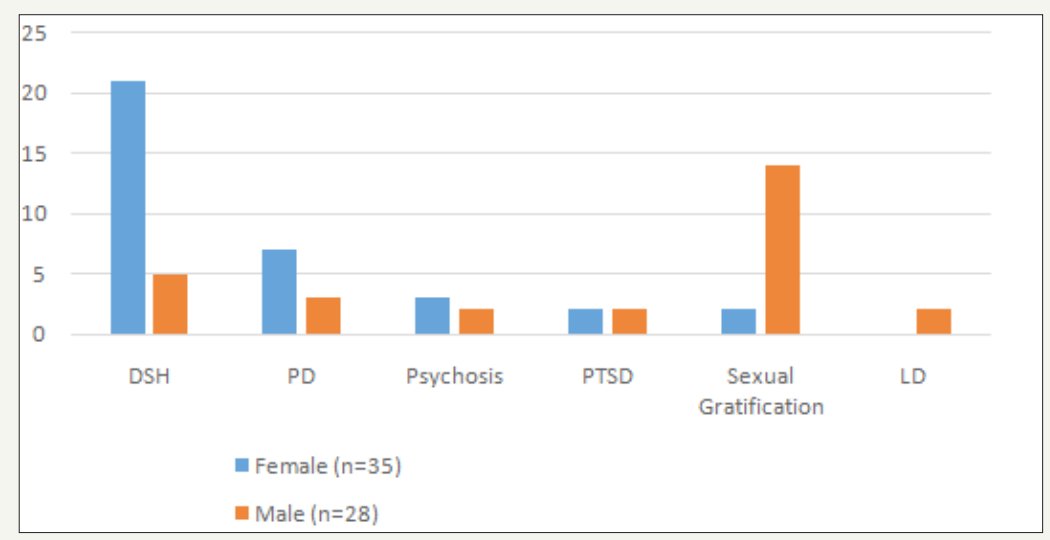

Figure 4: Underlying mental health illness.

Surgical and medical record and discharge letters from hospital admission were unable to classify sexual gratification and psychosis into underlying psychiatric illness and was found to be main short coming in understanding of psychiatric illness by medical doctors. The common sites of FB in patients with MHI were the oesophagus, stomach and subcutaneous tissue, shown in
Figure 4. We found female patients had a higher incidence of FB in the oesophagus (43\%) and skin (41\%), compared to male patients were they were more commonly found in the stomach (49\%) and rectum (31\%). We found that out of 257 admissions 84 (30\%) had no psychiatric diagnosis given on the discharge summary. The majority of these patients were those with FB in the rectum and 
urethra and these patients received no mental health assessment as part of their care. Table 3 shows the retrieval operation during this episode if we exclude the FB in subcutaneous tissue majority was removed endoscopically. The open removal by laparotomy was only $14 \%$ probably depending on mode of presentation i.e ingestion or insertion. There were a total of three deaths recoded, all with complications arising from FB ingestion, one was in the accidental group and two were in the MHI group. Those in the MHI group were both due to complications arising from repeated insertion of sharp objects subcutaneously leading to small bowel fistula Figure 5.
Table 3: Common operations for FB retrieval.

\begin{tabular}{|c|c|}
\hline Endoscopic GI & $88(34 \%)$ \\
\hline Endoscopic Urinary Track & $28(11 \%)$ \\
\hline EUA & $64(25 \%)$ \\
\hline Laparotomy & $36(14 \%)$ \\
\hline Surgical removal from S/C tissue & $28(11 \%)$ \\
\hline Endoscopic RT & $13(5 \%)$ \\
\hline
\end{tabular}

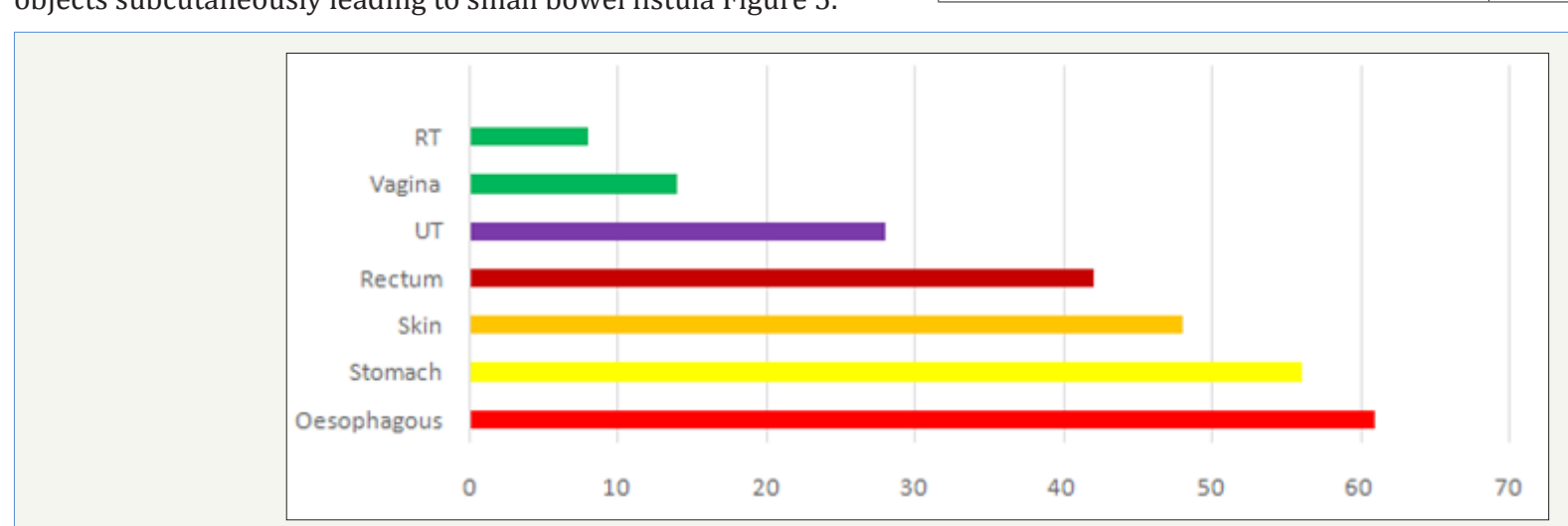

Figure 5: Most common sites of foreign bodies in patients with MHI ( $\mathrm{n}=257)$.

\section{Discussion}

Accidental FB ingestion is common in both extremes of life i.e children and in elderly people. Accidental FB is reported in children from 6 months to 6 years where they are exploring the world around them. As shown in our study the two extreme of accidental body were in children and elderly patients. While in intentional FB ingestion in patients with mental health problems are generally young adults who had various different diagnoses ranging from deliberate self-harm to personality disorders. This is slightly contrary to some of the medical literature where intentional FBs are reported more common in adulthood [3-4]. This is possibly due to separate child and adolescence mental health services in that area. FB insertion is a common presentation on surgical and gastroenterology wards. These patients can present to different surgical sub-specialities with different presentations i.e ingestion, insertion and introduction of FB in natural orifices for sexual gratification. The majority (90\%) of foreign bodies will pass spontaneously, with $10-20 \%$ requiring endoscopic intervention. According to literature only less than $1 \%$ of the patients with FB ingestion will need surgical removal of these FBs [5]. Most of the studies recommend endoscopic retrieval as procedure of choice and claims it to be successful in most patients [6] similar results are shown in our study where endoscopic procedures are successful in most of the patients.

There is limited medical literature on genital FB for sexual gratification and there management. Most of these patients are discharged with out and psychological consultation or help possibly due to patient embarrassment and or lack of medical health professionals in recognising this behaviour as a psychological problem. As shown in our study there is significant number of patients with genital FB and most of them were discharged without and help. Many patients with a MHI and recurrent FB are frequent visitors to the general surgical wards and are difficult to manage. As shown in our study their frequent presentation of mental health patients repeatedly. These patients should be referred to psychiatric services during their index admission for help with their, often complex needs. For the best outcomes and to reduce re-admission, these patients are best managed as part of a multidisciplinary team with all members having awareness and basic knowledge of management and treatment of this patient cohort [7].

There are many guidelines available for the management of FB ingestion in patients with or without MHI but there are no guidelines or criteria for referral of these patients to psychiatric services, although evidence suggests referral for those patients who have history of psychiatric problems [8] and for cases involving unusual foreign objects or those with a history of foreign object insertion [9]. However, psychiatric problems associated with insertion behaviour may go unidentified without routine psychiatric consultation, which has lead to the recommendation for prompt psychiatric evaluation for all patients who self-insert foreign objects [10-16].

\section{Conclusion}

Patient with FB ingestion are young patients with a significant underlying MHI. These patients should have an early psychiatric referral with the aim of establishing a diagnosis, to minimise harm to the patient during the hospital stay and to help reduce future presentations, where possible endoscopic approach should be adapted to minimise the effects of repeated anaesthesia which can lead to worsening of mental health illness especially during acute settings. 


\section{Acknowledgement}

A special thanks to Mr. Robert Jones, emergency theatre coordinator at Northampton General Hospital for help in patient identification and data collection.

\section{References}

1. Katsinelos P, Kountouras J, Paroutoglou G, Zavos C, Mimidis K (2006) Endoscopic techniques and management of foreign body ingestion and food bolus impaction in the upper gastrointestinal tract: a retrospective analysis of 139 cases. J Clin Gastroenterol 40(9): 784-789.

2. Lee JH, Kim HC, Yang DM, Kim SW, Jin W (2012) What is the role of plain radiography in patients with foreign bodies in the gastrointestinal tract? Clin imaging 36(5): 447-454.

3. Palese C, Kawas FH (2012) Repeat intentional foreign body ingestion: the importance of a multidisciplinary approach. Gastroenterol Hepatol (NY) 8(7): 485-486.

4. Chadda RK (2001) Psychiatry in non-psychiatric setting--a comparative study of physicians and surgeons. J Indian Med Assoc 99(1): 24-62.

5. Schenk C, Mugomba G, Dabidian RA, Scheuerecker H, Glaser F (2002) Laparoscopic extraction of a swallowed fork in a patient first diagnosed with bulimia nervosa. Surg endosc 16(2): 361.

6. Vishwanath G (2008) Laparoscopic Removal of Large and Sharp Foreign Bodies from the Stomach. Oman Med J 23(1): 42-45.
7. Caren Palese, Firas H, Al-Kawas FH (2012) Repeat intentional foreign body ingestion. The importance of a multidisciplinary approach. Gastroenterol Hepatol (NY) 8(7): 485-486.

8. Ayantunde AA, Oke T (2006) A review of gastrointestinal foreign bodies. Int J Clin Pract 60(6): 735-739.

9. Dakin WB (1948) Urological Oddities. Los Angeles, USA.

10. Frozanpour D (1976) foreign bodies in the bladder. Br J Clin Pract 30(5): 115-117.

11. Pattison EM, Kahan J (1983) The deliberate self-harm syndrome. The American Journal of Psychiatry 140(7): 867-872.

12. Rodham K, Hawton K, Evans E (2004) Reasons for deliberate self-harm: comparison of self-poisoners and self-cutters in a community sample of adolescents. Journal of the American Academy of Child and Adolescent Psychiatry 43(1): 80-87.

13. Kafka MP, Prentky RA (1997) Compulsive sexual behavior characteristics. Am J Psychiatry 154(11): 1632.

14. Bergner RM (2002) Sexual compulsion as attempted recovery from degradation: theory and therapy. J Sex Marital Ther 28(5): 373-387.

15. Bradford JM (1999) The paraphilias, obsessive compulsive spectrum disorder, and the treatment of sexually deviant behaviour. Psychiatr $Q$ 70(3): 209-219.

16. Bryant WR, Markham L, Kreipe RE (2010) Feeding and eating disorders in childhood. Int J Eat Disord 43(2): 98-111.
Creative Commons Attribution 4.0

International License

For possible submissions Click Here

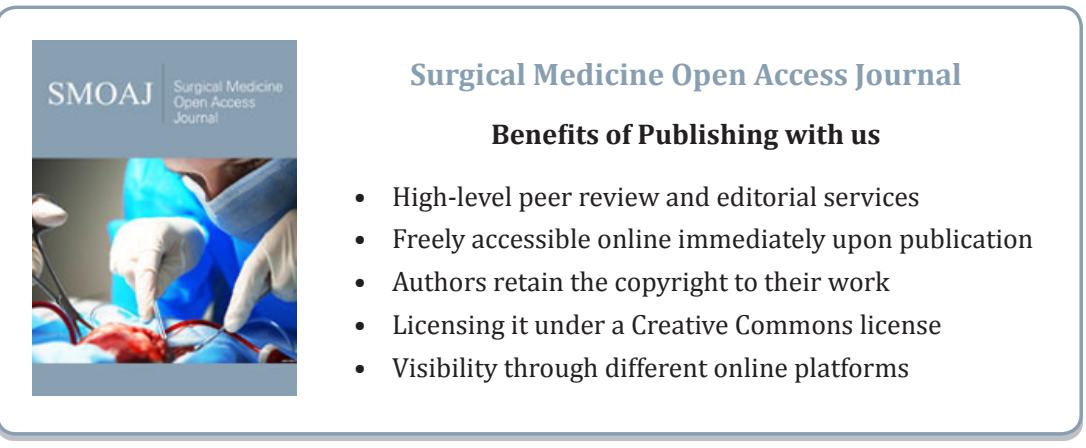

